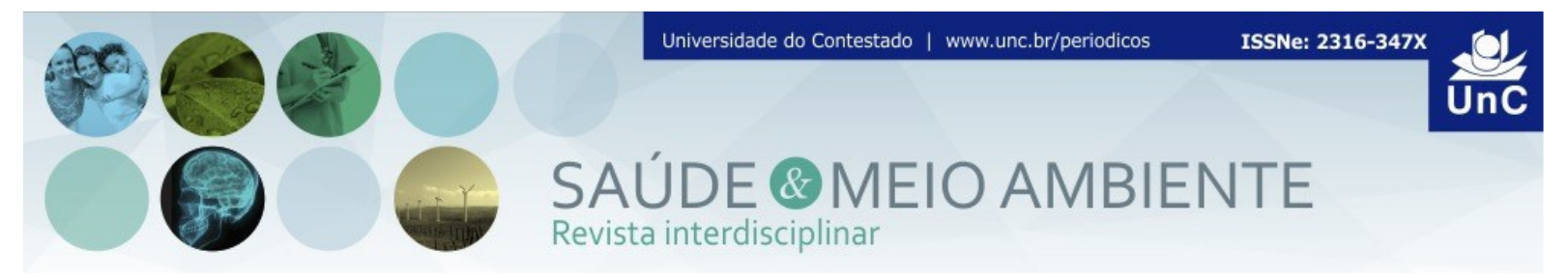

\title{
O CONHECIMENTO DE JOVENS FRENTE AS IST NO MUNICÍPIO DE NHAMUNDÁ - AM
}

\author{
Cleane Souza Duque ${ }^{1}$ \\ Luana Cardoso ${ }^{2}$ \\ Luiz Rodrigues Lima ${ }^{3}$ \\ João Viriato Mazalo ${ }^{4}$ \\ Bruno Mori ${ }^{5}$
}

\begin{abstract}
RESUMO
Os adolescentes estão iniciando sua vida sexual cada vez mais cedo. A adolescência é uma fase caracterizada pela faixa etária de 12 a 18 anos, repleta de descobertas e vulnerabilidades, onde ocorre à expressão própria de escolha individual e questionamentos perante a sociedade, relacionado ao conhecimento do próprio corpo, relações sexuais, uso de drogas e consequentemente exposição às infecções sexualmente transmissíveis (IST). Neste contexto, idealizou-se este estudo com o objetivo de avaliar o grau de conhecimento dos jovens frente às Infecções Sexualmente Transmissíveis (IST) no município de Nhamundá/Am. Tratase de um estudo descritivo, observacional de caráter quantitativo com amostra de 40 (quarenta) adolescentes regularmente matriculados no $8^{\circ}$ e $9^{\circ}$ ano do ensino fundamental da Escola Estadual Professor Gilberto Mestrinho, no período entre agosto a setembro de 2015. Os dados foram coletados com auxílio de questionário sobre o conhecimento dos jovens em relação à sexualidade, e utilizou-se a estatística descritiva para análise dos dados. Nos resultados constatou-se que dos 40 adolescentes participantes do estudo, quando questionados se conheciam os métodos de prevenção das IST, a opção respondida por $70 \%$ (28) dos alunos foi de: não possuir conhecimentos e apenas 30\% (12) alunos responderam: possuir conhecimentos sobre a prevenção e que, entretanto, a falta do diálogo com os pais sobre a vida sexual, foi apontado como o principal motivo de desconhecimento sobre às IST $p<0.09172^{*}$. Assim, conclui-se que existe ausência de conhecimentos básicos e indispensáveis para prevenir as IST na maioria dos alunos que participaram da entrevista, resultante da falta de diálogo familiar, educação sexual ou até mesmo timidez do próprio adolescente em abordar o assunto.
\end{abstract}

Palavras-chaves: Adolescente. Doenças sexualmente transmissíveis. Saúde do adolescente.

\footnotetext{
1Enfermeira, Faculdade Estácio do Amazonas. Brasil. E-mail: cleaneduque@gmail.com

2Enfermeira, Faculdade Estácio do Amazonas, Especialista em Saúde Pública. Brasil. E-mail: luanaoliveira147@gmail.com

3 Nutricionista. Mestrando em Imunologia Básica e Aplicada. Universidade Federal do Amazonas (UFAM PPGIBA). Especialista em Saúde Pública. Amazonas. Brasil. E-mail: eduardordgznutri@gmail.com

${ }^{4}$ Mestrando do Programa de Pós-graduação em Ciências da Saúde da Universidade Federal do Amazonas (UFAM) Brasil. Graduado em Optometria com ênfase em Saúde Pública pela Universidade Lúrio. Docente no Curso de Licenciatura em Optometria na Universidade Lúrio em Moçambique, campus Marrere, Nampula. Moçambique. E-mail: joaomazalo@gmail.com

${ }^{5}$ Mestre em Biologia Urbana/UNL, Doutorando em Imunologia Básica e Aplicada, Universidade Federal do Amazonas (PPGIBA-UFAM). Amazonas. Brasil. E-mail: bruno.mori@ufam.edu.br
} 


\title{
THE YOUTH KNOWLEDGE FACE TO STI IN THE CITY COUNCIL OF NHAMUNDÁ- AM
}

\begin{abstract}
Teenagers are starting their sex lives earlier and earlier. Adolescence is a phase characterized by the age group of 12 to 18 years old, full of discoveries and vulnerabilities, where there is the expression of individual choice and questions before society, related to the knowledge of one's own body, sexual relations, drug use and consequently exposure to sexually transmitted infections (STIs). In this context, this study was idealized with the objective of evaluating the degree of knowledge of young people regarding Sexually Transmitted Infections (STIs) in the city council of Nhamundá / Am. This is a descriptive, observational study of a quantitative character with a sample of 40 (forty) teenagers regularly enrolled in the 8th and 9th grade of elementary school at Escola Estadual Professor Gilberto Mestrinho, in the period between August and September 2015. The data were collected through questionnaire on the knowledge of young people in relation to sexuality, and descriptive statistics were used for data analysis. In the results it was found that of the 40 adolescents participating in the study, when asked if they knew the methods of STI prevention, the option answered by $70 \%(28)$ of the students was: not having knowledge and only $30 \%(12)$ students answered: having knowledge about prevention, however, the lack of dialogue with parents about sexual life, was pointed out as the main reason for not knowing about STIs $p<0.09172 *$. Thus, it is concluded that there is a lack of basic and indispensable knowledge to prevent STIs in the majority of students who participated in the interview, resulting from the lack of family dialogue, sexual education or even shyness of the adolescent himself in addressing the subject.
\end{abstract}

Keywords: Adolescent. Sexually transmitted diseases. Adolescent health.

\section{INTRODUÇÃO}

Adolescência é uma fase de crescimento marcada por transformação entre a infância e a idade adulta, que compreende a idade de 12 a 18 anos, com intensas mudanças fisiológicas, psicológicas que afetam a interação social. Esse processo de amadurecimento ocorre de forma muito rápida, e é importante para a formação do indivíduo, bem como para sua inserção e afirmação no meio social ${ }^{(1)}$. Nesse período, o adolesceste se debate com a necessidade de busca da sua própria identidade, como forma de se estabelecer perante a sociedade, num momento que pode ser considerado um dos mais importantes na sua formação psicossocial(2).

As vulnerabilidades e fragilidades que advém da fase da adolescência, devese a falta de experiências adquiridas no decorrer da vida, bem como da ausência de apoio e de diálogo perante situações relacionadas a modificações anatômicas, 
ligadas ao conhecimento do próprio corpo, relações sexuais, uso de drogas e consequentemente exposição às infecções sexualmente transmissíveis $(\text { IST })^{(3),(4)}$.

A curiosidade aliada ao prazer da descoberta fazem com que muitos adolescentes tenham intenção de criar novas experiências emocionais, amorosas e corporais, que acabam marcando essa intensa relação entre ambos os sexos, culminado com a primeira relação sexual e consequente exposição as IST, colocando em risco à sua saúde, pois nem sempre os métodos de prevenção são utilizados, uma vez que nessa fase o conhecimento sobre as IST é escasso ${ }^{(5)}$. A ausência de habilidades em tomar decisões junto com a falta de experiência de ambos representam uma condição de vulnerabilidade às IST/AIDS(6),(7). Quando aborda-se sobre as Infecções Sexualmente Transmissíveis (IST) no meio dos adolescentes e até de alguns jovens nota se um grande grau de desconhecimento no assunto, o que se supõe que existe um número elevado de adolescentes que praticam relação sexual desprotegidas, quer seja por falta de conhecimento e/ou por negligencia, o que faz acreditar que os números de casos de IST podem ser bem maiores do que os divulgados ${ }^{(1)}$.

As infecções sexualmente transmissíveis (IST) são transmitidas principalmente por contato sexual desprotegido, ou seja, sem o uso de camisinha, com uma pessoa que esteja infectada. Também podem ser transmitidas por feridas, corrimentos, objetos cortantes, sendo no seu estágio inicial muitas delas difícil de identificar porque são assintomáticas, portanto, é recomendado que a pessoa que manteve relação sexual sem camisinha procure o serviço de saúde ${ }^{(7),(8)}$.

$\mathrm{Na}$ prevenção das Infecções Sexualmente Transmissíveis (IST) nos adolescentes, é importante realçar que todos os indivíduos envolvidos na educação e na formação dos adolescentes, devem desempenhar seu papel, quer o professor, quer os encarregados, quer a sociedade, criando espaço de diálogo aberto, dando a possibilidade dos adolescentes estarem dotados de conhecimentos para se prevenir $^{(9),(10)}$. No Brasil, as Infecções Sexualmente Transmissíveis (IST) estão a nível da atenção primária afetando homens e mulheres. Como forma de minimizar o impacto das Infecções Sexualmente Transmissíveis (IST), tem se desenvolvido ações de prevenção através de palestras e oficinas, com a finalidade de consciencializar as pessoas da importância do uso da camisinha, para evitar a contaminação(11)(6).

Vários profissionais da saúde trabalham em programas que tem como objetivo intensificar o uso de preservativos, principalmente entre os adolescentes, tendo em vista que esse grupo possui um alto índice de exposição. A relevância destes programas no estado de saúde dos adolescentes, tem surtido grandes resultados, razão pela qual é necessário disseminar informações que podem ser usados no combate às Infecções Sexualmente Transmissíveis (IST). Neste contexto, idealizou-se este estudo com objetivo de avaliar o grau de conhecimento dos jovens frente às Infecções Sexualmente Transmissíveis (IST) no município de Nhamundá/Amazonas. 


\section{METODOLOGIA}

Trata-se de um estudo descritivo, observacional de caráter quantitativo realizado por meio da aplicação de questionário, onde foram incluídos adolescentes regularmente matriculados com idade de 12 a 15 anos de ambos os sexos, escolhidos de forma aleatória, totalizando uma amostra de 40 alunos da Escola Estadual Professor Gilberto Mestrinho, sob a direção da professora Lia Costa Nascimento, no município de Nhamundá (Am), durante os meses de agosto a setembro de 2015.

O município de Nhamundá no Amazonas, possui uma área territorial de $14.105,590 \mathrm{~km}^{2}$, com população estimada de 18.278 habitantes, considerado o trigésimo quinto município mais populoso do estado do Amazonas sendo localizado na zona geográfica do Baixo Amazonas (12).

Esta pesquisa foi aprovada pelo Comitê de Ética da Faculdade Estácio do Amazonas com o $\mathrm{n}^{\circ} 1.235 .751$.

Após a coleta de dados, os resultados foram colocados em planilha do Microsoft Excel $2010^{\circledR}$. Posteriormente, as relações entre as variáveis estudadas foram desenvolvidas com base em suas frequências absolutas. As análises de significância foram dadas pelos testes de Qui-Quadrado. Para todos os testes foi usado um nível de significância de 0,05.

O questionário foi elaborado por $\mathrm{Genz}^{(8)}$ e adaptado pelos pesquisadores deste estudo. Constituído de 26 perguntas de múltipla escolha, respondidas pelos próprios entrevistados, abordando tópicos básicos sobre Infecções Sexualmente Transmissíveis e as formas de contágio e prevenção. Ao final, contém perguntas sobre o perfil e o comportamento sexual do entrevistado, de forma anônima. Os pais/responsáveis dos adolescentes receberam leram e assinaram previamente o Termo de Consentimento Livre e Esclarecido em duas cópias autorizando a participação dos menores, e os adolescentes participantes da pesquisa receberam o Termo de Assentimento Livre e Esclarecido em duas cópias, para ler e, após concordar, assinar as duas vias e então responder ao questionário. Uma das vias ficou com o participante da pesquisa e a outra com os pesquisadores.

\section{RESULTADOS}

Participaram da pesquisa $20 \%$ dos 196 alunos do oitavo e nono ano, escolhidos de forma aleatória, totalizando 40 alunos, 20 do sexo feminino e 20 do sexo masculino, com idades entre 12 a 15 anos, todos estudantes da Escola Estadual Professor Gilberto Mestrinho do Município de Nhamundá - AM. 
Gráfico 1 - Conhecimento dos participantes sobre a prevenção em relação às IST.

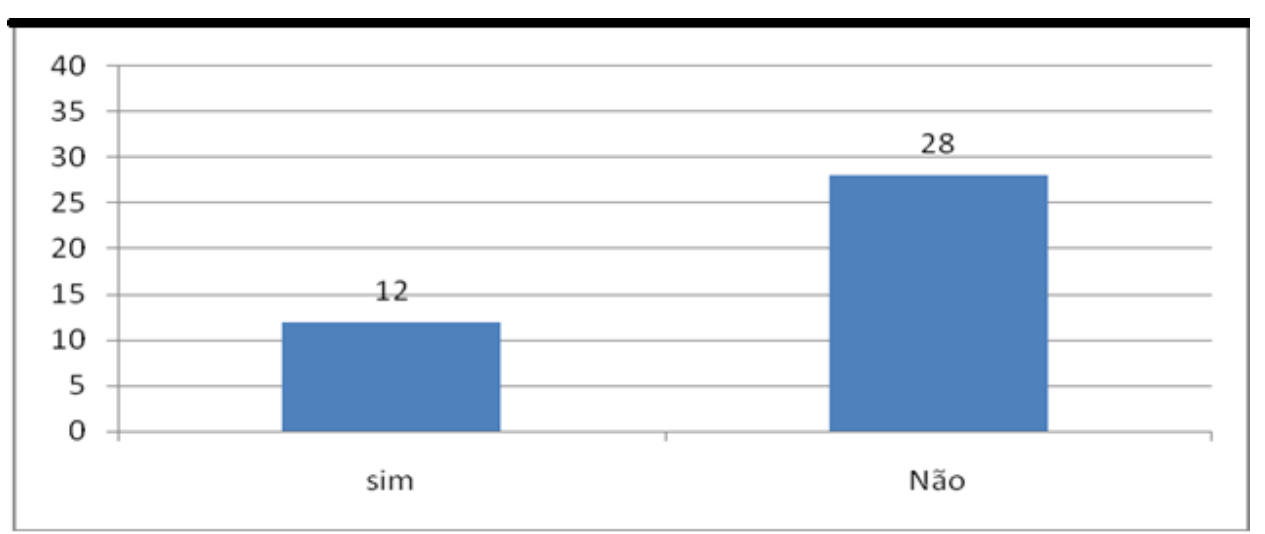

Fonte: Pesquisadores (2015)

Após a realização do questionário, observou-se que $70 \%$ (28) dos alunos responderam que não tinham conhecimentos sobre a prevenção das IST e apenas $30 \%$ (12) alunos responderam que sim (Gráfico 1).

Tabela 1 - Respostas dos adolescentes sobre cuidados e conhecimentos sobre as IST.

\begin{tabular}{lcccccccc}
\hline & \multicolumn{3}{c}{ SIM } & \multicolumn{5}{c}{ NÃO } \\
\cline { 2 - 8 } & Mas & Fem & $\%$ & Mas & Fem & $\%$ & p-value \\
\hline Diálogo com os pais sobre a vida sexual & 2 & 4 & 15 & 18 & 16 & 85 & 0.09172 \\
UBS para tirar dúvidas & 5 & 2 & 17.5 & 15 & 18 & 82.5 & 0.08062 \\
Todas as ISTs tem cura & 6 & 6 & 30 & 10 & 18 & 70 & 0.01822 \\
AIDS tem cura & 6 & 6 & 30 & 10 & 18 & 70 & 0.01822 \\
Aumento da incidência de IST & 19 & 19 & 95 & 1 & 1 & 5 & 0.15 \\
Conhecimento em relação às IST & 13 & 18 & 77.5 & 7 & 2 & 22.5 & 0.06421 \\
\hline
\end{tabular}

Fonte: Pesquisadores (2015)

Dos 40 entrevistados, 85\% (34) alunos responderam que não há liberdade para conversar sobre sexo com seus pais e $15 \%$ (6) responderam que sim (Tabela 1).

Quando questionados sobre a busca as Unidades Básicas de Saúde (UBS) para tirarem dúvidas sobre suas mudanças fisiológicas, $82,5 \%$ (33) alunos responderam não e 17,5\% (7) responderam que sim (Tabela 1). 
Quando perguntados se achavam que todas as IST possuem cura, 70\% (28) alunos responderam não e $30 \%$ (12) sim (Tabela 1).

Constou-se que, $70 \%$ (28) alunos responderam que a AIDS não possui cura e $30 \%$ (12) responderam que sim (Tabela 1).

Quando inqueridos sobre a incidência de IST está aumentando, 95\% (38) alunos responderam que achavam que sim e $5 \%$ (2) não (Tabela 1).

Outra questão que foi levantada, foi a respeito do conhecimento sobre as IST, no total $77,5 \%$ (31) alunos responderam que possuíam conhecimento em relação as IST 22,5 (9) responderam não possuir conhecimento (Tabela 1), reforçando a ideia que esse tema tem sido discutido em algum momento da vida do adolescente, seja em casa, rua ou na escola.

Tabela 2 - Pessoas com quem os adolescentes esclareciam suas dúvidas e prevenção as IST.

\begin{tabular}{|c|c|c|c|c|c|c|c|c|c|}
\hline \multicolumn{3}{|c|}{ Mídia } & \multicolumn{3}{|c|}{ Escola } & \multicolumn{3}{|c|}{ Amigos } & \multirow[b]{2}{*}{$p$-value } \\
\hline Mas. & Fem. & $\%$ & Mas. & Fem. & $\%$ & Mas. & Fem. & $\%$ & \\
\hline & & & & & & & & & 0.00935 \\
\hline \multirow[t]{4}{*}{4} & 6 & 25 & 5 & 2 & 17.5 & 11 & 12 & 57.4 & 8 \\
\hline & & \multicolumn{3}{|c|}{ Anticoncepcional } & \multicolumn{3}{|c|}{ Camisinha } & & \\
\hline & & Mas & Fem. & $\%$ & Mas. & Fem. & $\%$ & & \\
\hline & & 0 & 1 & 2.5 & 20 & 19 & 97.5 & & 0.1662 \\
\hline
\end{tabular}

Fonte: Pesquisadores (2015)

Os adolescentes relataram que conversavam com os amigos sobre sexualidade $57,4 \%$ (23), 25\% (10) através da mídia e 17,5\% (7) na escola (Tabela 2).

Quanto à melhor forma de evitar as IST, os adolescentes responderam que é com o uso de camisinha opção respondida por $97,5 \%$ (39) alunos e apenas 2,5\% (1) respondeu anticoncepcionais. (Tabela 2).

\section{DISCUSSÃO}

Para os adolescentes terem informações sobre a prevenção das IST, os profissionais necessitam de estar dotados de conhecimentos e aperfeiçoar a melhor maneira de falar em relação à saúde do adolescente, para que possam trabalhar junto à escola, criando estratégias de maneira que chegue uma informação 
concreta, respeitando seus direitos, permitindo mostrar capacidade de agir com responsabilidade juntamente com a família e a equipe de saúde ${ }^{(13-15)}$.

A família é fundamental no desenvolvimento do adolescente, o respeito e a compreensão faz com que o mesmo expresse seus sentimentos, guiando no caminho que melhor lhe cabe estando em processo de transformação e aceitação. $O$ relacionamento entre pais e filhos do mesmo sexo, é uma importante estratégia no processo de identificação de gênero nessa fase da vida(15-17).

$\mathrm{Na}$ puberdade, ocorrem transformações físicas e sexuais, que alteram rapidamente a autoimagem dos adolescentes, fazendo com que os familiares fiquem confusos e ansiosos quando o adolescente começa a ter mudanças no corpo ou adquirirem interesses sexuais evidentes ${ }^{(18),(19) .}$

No que diz respeito as dúvidas sobre a sexualidade, os resultados são compatíveis com o estudo de realizado por Torquato (2017), no qual participaram alunos com idades entre 14 e 17 anos, sendo 13 (52\%) do sexo feminino e 12 (48\%) do sexo masculino, onde constatou-se que, para tirar as dúvidas sobre sexualidade, $7(28 \%)$ nunca esclareceram suas dúvidas, $5(20 \%)$ perguntam para os pais, $2(8 \%)$ com os amigos, $4(16 \%)$ com o unidade de saúde, $4(16 \%)$ conversam com o namorado e $1(4 \%)$ tiraram suas dúvidas na internet, onde nota se que o diálogo com os pais e encarregados de educação, bem como com a unidade de saúde, está baixo em ambos os casos $^{(20)}$.

As IST são consideradas um grave problema de saúde pública, os infectados não buscam informações para o tratamento ou quando decidem buscar tais informações, a IST já está em estágio avançado. É importante realçar, que o diagnóstico precoce é fundamental para o tratamento, acompanhamento e prognóstico das IST, apesar de algumas delas não possuir cura, como é o caso da AIDS. (1),(2).

A falta de conhecimento sobre a AIDS, deve-se a não consciência da comunidade na necessidade de dotar os adolescentes de conhecimentos que podem e devem ser usados para evitar a transmissão e o aumento de casos de infecções relacionados a AIDS, tendo em conta que essa faixa é vulnerável, tornando-os portadores de informações que vão ajudar a prevenir da AIDS, uma IST que até hoje não tem cura(20), (21).

No que concerne a fonte de obtenção de conhecimento sobre as IST, os resultados do estudo são contraditórios com o estudo realizado por Padilha (2015) numa escola pública estadual de um bairro de periferia da cidade de Guarapuava, em 207 adolescentes (116 do sexo feminino e 91 do sexo masculino), entre 15 e 21 anos de idade, onde constatou-se que $25 \%$ dos jovens afirmaram ter adquirido conhecimento em casa com os pais; $7 \%$ através de livros e revistas; $8 \%$ através dos amigos; $31 \%$ através da escola; $56 \%$ através da televisão e $60 \%$ através de serviços de saúde. Essa contradição deve se pelo fato de no estudo realizado por Padilha (2015), os jovens podiam escolher mais de uma alternativa para a pergunta; assim, a percentagem refere-se às respostas e não ao número de adolescentes ${ }^{(22)}$. 
Em relação ao método de evitar as IST, o resultado do estudo é compatível com a pesquisa realizada por Rodrigues (2015), numa escola da rede pública de ensino no município de São Paulo em adolescentes com idade entre 15 e 17 anos que estavam matriculados regularmente, observou-se, que todos os adolescentes afirmaram conhecer algum tipo de método de prevenção $(100,0 \%)$, sendo o uso de preservativo $(100,0 \%)$ citado por todos os estudantes ${ }^{(23)}$.

\section{CONCLUSÃO}

Após a realização do estudo conclui-se que existe ausência de conhecimentos básicos e indispensáveis para prevenir às Infecções Sexualmente Transmissíveis (IST) na maior parte dos alunos que participaram da entrevista, resultante da falta de diálogo familiar, educação sexual ou até mesmo timidez do próprio adolescente em abordar o assunto, tornando-se incapazes de fazer escolhas certas. Deste modo, torna se fundamental à criação de programas que incentivam as famílias a falarem sobre a educação sexual, a realização de estratégias que possam lograr êxitos de forma eficaz, como atividades de conscientização e palestras para o público adolescente, para que possam ser esclarecidas e tiradas dúvidas sobre às Infecções Sexualmente Transmissíveis (IST) no município de Nhamundá/Amazonas.

É importante realçar que a participação da família na formação dos adolescentes é crucial para a saúde mental, social, bem como para a prevenção de aparecimento das Infecções Sexualmente Transmissíveis (IST) e da gravidez precoce, de modo a acompanhar de perto essa fase repleta de descobertas e curiosidades.

Este estudo foi realizado com uma amostra relativamente baixa, talvez esses dados não expressem o que de fato ocorre em outras localidades, reforçando a ideia da necessidade de realização de futuros estudos mais aprofundados para avaliar melhor o conhecimento dos jovens frente às Infecções Sexualmente Transmissíveis (IST) no Brasil.

\section{REFERÊNCIAS}

1. Rosa LM, Nascimento AAC, Dias ALF, Pereira MFR, Mota MA, Melo Filho PR, et al. Promoção da saúde na escola: prevenção da gravidez e de infecções sexualmente transmissíveis. Brazilian J Heal Rev [Internet]. 2020;3(1):706-16. Available from: http://www.brjd.com.br/index.php/BJHR/article/view/6580/5793

2. Coutinho RX, Santos WM dos, Folmer V, Puntel RL. Prevalência de comportamentos de risco em adolescentes Risk behaviors prevalence in adolescents. Cad Saúde Colet. 2013;21(4):441-9. 
3. Carvalho O, Pinto S. Conhecimento sobre as infecções sexualmente transmissíveis por estudantes adolescentes de escolas públicas. Adolesc Saude ,Rio Janeiro,. 2018;15:7-17.

4. Gerais M, Aparecida L, Chagas A, Gerais M, Campos- PHA, Costa RA, et al. A importância do conhecimento sobre Infecções Sexualmente Transmissíveis ( IST ) pelos adolescentes e a necessidade de uma abordagem que vá além das concepções biológicas. Rev Em Extensão. 2019;18:63-80.

5. Fiedler MW, Araújo A, Souza MCC de. The prevention of teenage pregnancy in adolescent's view. Texto Context - Enferm [Internet]. 2015 Mar;24(1):30-7. Available from: http://dx.doi.org/10.1590/0104-07072015000130014

6. Ministério da Saúde. Brasil. Secretaria de Vigilância em Saúde. Departamento de Vigilância, Prevenção e Controle das Infecções Sexualmente Transmissíveis do $\mathrm{H}$ e das HV. Boletim Epidemiológico HIVIAids. HIV Aids Bol Epidemiológico [Internet]. 2017;64. Available from: http://www.aids.gov.br/pt-br/pub/2018/boletimepidemiologico-hivaids-2018

7. Viera PM, Matsukura TS. Modelos de educação sexual na escola: concepções e práticas de professores do ensino fundamental da rede pública. Rev Bras Educ [Internet]. 2017 Jun;22(69):453-74. Available from: http://dx.doi.org/10.1590/S1413-24782017226923\%0AModelos

8. Genz N, Maria S, Meincke K, Laura M, Carret V, Cândida A, et al. Sexually Trasmited Diseases: Knowledge and Sexual Behavior of Adolescents. Texto Context Enferm [Internet]. 2017;26(2):1-12. Available from: http://dx.doi.org/10.1590/0104-07072017005100015.

9. Willis LA, Kachur R, Castellanos TJ, Spikes P, Gaul ZJ, Gamayo AC, et al. Developing a Motion Comic for HIVISTD Prevention for Young People Ages 1524, Part 1: Listening to Your Target Audience. Health Commun [Internet]. 2018 Feb 21;33(2):212-21. Available from: http://dx.doi.org/10.1080/10410236.2016.1255840

10. Sperhacke RD, Rapone L, Kato SK. HIV prevalence and sexual behavior among young male conscripts in the Brazilian army, 2016. Medicine (Baltimore) [Internet]. 2018;0:0-6. Available from: http://dx.doi.org/10.1097/MD.0000000000009014

11. Bastos FI, Bastos LS, Coutinho C, Toledo L, Velasco-de-castro CA, Sperandei S, et al. HIV, HCV, HBV, and syphilis among transgender women from Brazil. Medicine (Baltimore). 2018;1(October 2017):16-24.

12. Instituto Brasileiro de Geografia e Estatística. Amazonas em Mapas. 2015.

13. Silva JN da, Cabral JF, Nascimento VF do, Lucietto GC. Impactos do Diagnostico da Infeccao Sexualmente Transmissivel na Vida da Mulher. Enferm em foco. 2018;9(2):23-7. 
14. Newton-Levinson A, Leichliter JS, Chandra-Mouli V. Help and Care Seeking for Sexually Transmitted Infections Among Youth in Low- and Middle-Income Countries. Sex Transm Dis [Internet]. 2017 Jun;44(6):319-28. Available from: http://insights.ovid.com/crossref?an=00007435-201706000-00001

15. Pereira J de CN, Barbosa LU, Henriques AHB, Araújo PM de OA, Muniz MLC, Melo EC dos $S$, et al. Educação em saúde com adolescentes escolares acerca da sexualidade e infecções sexualmente transmissíveis: um relato de experiência. Rev Eletrônica Acervo Saúde [Internet]. 2019 Aug 31;29(29):e1130. Available from: https://acervomais.com.br/index.php/saude/article/view/1130

16. Burlamaqui JCF, Cassanti AC, Borim GB, Damrose E, Villa LL, Silva L. Human Papillomavirus and students in Brazil: an assessment of knowledge of a common infection - preliminary report. Braz J Otorhinolaryngol [Internet]. 2017 Mar;83(2):120-5. Available from: http://dx.doi.org/10.1016/j.bjorl.2016.02.006

17. Rosa S, Santos S. Assistencia do Enfermeiro na Prevencao do HPV. Rev Inic Cient e Ext. 2018;1(1):28-31.

18. Santos SB dos, Machado APDA, Sampaio LA, Abreu LC De, Bezerra IMP. Sífilis adquirida: construção e validação de tecnologia educativa para adolescentes. J Hum Growth Dev [Internet]. 2019 May 6;29(1):65-74. Available from: http://dx.doi.org/10.7322/jhgd.157752

19. Bezerra IMP, Sorpreso ICE. Concepts and movements in health promotion to guide educational practices. J Hum Growth Dev [Internet]. 2016 Apr 28;26(1):11. Available from: http://www.revistas.usp.br/jhgd/article/view/113709

20. Torquato, Bianca Gonçalves Silva et al. O saber sexual na adolescência. Rev Ciênc Ext. 2017;13(3):54-63.

21. Cho $\mathrm{Y}-\mathrm{H}$, Span SA. The effect of alcohol on sexual risk-taking among young men and women. Addict Behav [Internet]. 2010 Aug;35(8):779-85. Available from: https://doi.org/10.1016/j.addbeh.2010.03.007

22. Padilha, Ana Paula et al. O conhecimento de adolescentes sobre doenças sexualmente transmissíveis. Rev Eletrônica Gestão Saúde. 2015;6:2249-60.

23. Rodrigues MO, Onofre PS de C, Oliveira PP, Amaral JL. Conhecimento Dos Adolescentes De Uma Escola Da Rede Pública Sobre As Principais Doenças Sexualmente Transmissíveis. Rev Enferm do Cent Oeste Min - RECOM. 2015;3(4):1268-80. 